\title{
Protective Effect of Natural Flavonoid Quercetin Against Exercise-induced Oxidative Damage in Experimental Rat Model
}

\author{
Zhang Wei-qiang ${ }^{\mathrm{a}}$ \\ Teaching and Research Department of P.E. Central South University, Changsha City, Hunan \\ Province, 410083, Chinay \\ azhangweiznqiang@sina.com
}

Keywords: quercetin; oxidative damage; exhaustive treadmill exercise; rat Abstract. This study was conducted to investigate the effect of natural flavonoid quercetin on exercise-induced oxidative damage. Twenty-eight rats were randomly divided into four groups, a control and three quercetin administered groups (20, 40 and $60 \mathrm{mg} / \mathrm{kg}$ ). Quercetin was orally administered to rats once a day for 28 days, followed by the exhaustive treadmill exercise. The results show that quercetin prolonged the running time to exhaustion of rats, as well as decreasing the levels of lactate dehydrogenase and creatine kinase in serun, and the levels of myeloperoxidase, malondialdehyde and 8-hydroxy-2'-deoxyguanosine in liver and muscle. This indicated that quercetin has a protective effect on exercise-induced oxidative damage.

\section{Introduction}

Quercetin (3,3',4',5,7-pentahydroxyflavone, Fig. 1), which belongs to the flavonol class of flavonoids, a type of plant-based chemical, contains the basic flavonoid structure of 15 carbon atoms arranged in 3 rings $\left(\mathrm{C}_{6}-\mathrm{C}_{3}-\mathrm{C}_{6}\right)$ [1]. It is present in high concentrations in fruits and vegetables such as tea, apples, mulberries, onions, potatoes, broccoli, soybeans, peanuts, and red wine [2]. Recent studies have showed that quercetin has multiple biological activities, including cardioprotection, cataract prevention, neuroprotective, antioxidative, anti-cancer, anti-ulcer, anti-inflammatory, anti-allergic, antiviral, anti-apoptosis and anti-bacterial activities [3]. Especially, quercetin is apotent oxygen free radical scavenger and shows potent scavenger activity against superoxide and hydroxyl radicals, nitric oxide and also peroxynitrite [4], which suggesting that it might be beneficial to exercise-induced oxidative damage. Therefore, the present study was to explore the potential protective effects of quercetin on exercise-induced oxidative damage in male rats.

\section{Materials and Methods}

Chemicals and reagents. Quercetin ( > 98\% purity) was purchased from Yiji Industrial Co., Ltd. (Shanghai, China). The detection kit for lactate dehydrogenase (LDH) was purchased from SenBeiJia Biological Technology Co., Ltd. (Nanjing, China). The detection kits for creatine kinase (CK), myeloperoxidase ( (MPO) and malondialdehyde (MDA) were purchased from Jiancheng Bioengineering Institute (Nanjing, China). The enzyme-linked immunosorbent detection kit for 8-hydroxy-2'-deoxyguanosine (8-OHdG) was purchased from (Japan Institute for the Control of Aging (Fukuroi, Japan). All other chemicals and reagents were of the highest quality commercially available.

Experimental animals. Male Wistar rats, weighing $220 \pm 10$ g, were obtained from the laboratory animal center of Hunan (Changsha, China). The rats had free access to commercial food and tap water, and housed in a specific pathogen-free environment at $23 \pm 2{ }^{\circ} \mathrm{C}$, under a 12-12-h light-dark cycle. All experimental procedures were in accordance with guidelines for the care and use of laboratory animals were approved by the Ethics Committee of Central South University (Changsha, China).

Experimental grouping and treatment. After 7 days adaptive feeding, thirty-two rats were chosen and randomly divided into four groups: namely, control (C) group, low-dose quercetin (LQ) group, medium-dose quercetin (MQ) group, and high-dose quercetin (HQ) group. The rats of $\mathrm{C}$ group was administered with $2.0 \mathrm{~mL}$ of corn oil by gavage once a day for 28 days, while the rats of quercetin 
(LQ ,MQ, and HQ) groups received various doses of quercetin (20, 40 and $60 \mathrm{mg} / \mathrm{kg}$ body weight, suspended in $2.0 \mathrm{~mL}$ of corn oil) by gavage once a day for 28 day.

Exhaustive treadmill exercise. Exhaustive treadmill exercise was performed as described previously with some modifications [5]. The rats of all groups were practiced to adapt to the treadmill exerciser (ZH-PT, Zhenghua biological instrument equipment Co., Ltd, Anhui, China) duration for 3 days at a speed of $10 \mathrm{~m} / \mathrm{min}$ for $10 \mathrm{~min}$ and a slope of $0^{\circ}$ before the exhaustive treadmill exercise. On the 28 days, the rats were made to carry out the exercise until exhausted based on the schedule: first-level load: $0^{\circ}, 8.2 \mathrm{~m} / \mathrm{min}, 15 \mathrm{~min}$; second-level load: $5^{\circ}, 15 \mathrm{~m} / \mathrm{min}, 15 \mathrm{~m}$; third-level load: $10^{\circ}$, $19.3 \mathrm{~m} / \mathrm{min}$, until exhausted. Exhaustion was determined as the rat being unable to upright itself when placed on its back, and the mean running time to exhaustion was recorded.

Analysis of biochemical parameters. The rats were anesthetized with pentobarbital sodium (5 $\mathrm{mg} / 100 \mathrm{~g}$ body weight, i.p.), and sacrificed immediately after the exhaustive treadmill exercise. Blood samples were collected from the abdominal aorta and then serum was immediately separated by centrifugation at 3,000 $\times \mathrm{g}$ at room temperature for $10 \mathrm{~min}$ for the assay of LDH, CK. After the blood was collected, the liver and gastrocnemius muscle were immediately dissected, frozen in liquid nitrogen, and kept at $-80{ }^{\circ} \mathrm{C}$ for the assay of MPO, MDA and 8-OHdG. All of the biochemical parameters were determined by using commercial kits following the manufacturer's recommended protocol.

Statistical analysis. All data were expressed as means \pm SD. SPSS software for Windows version 17.0 (SPSS Inc., Chicago, IL) was used for statistical analysis. The difference between test and controls were evaluated by Student's t-test. Differences were considered to be statistically significant when $\mathrm{p}<0.05$.

\section{Results and Discussion}

Effects of quercetin on running time to exhaustion of rats. It has been previously demonstrated that exhaustive treadmill exercise is an experimental exercise model which works well for evaluating the endurance capacity of rats and gives a high reproducibility [6]. As shown in Fig. 2, the running time to exhaustion in the LQ, MQ and HQ groups were significantly prolonged than that of the C (control) group $(\mathrm{p}<0.05)$. This data clearly reveal that quercetin could enhance exercise endurance capacity.

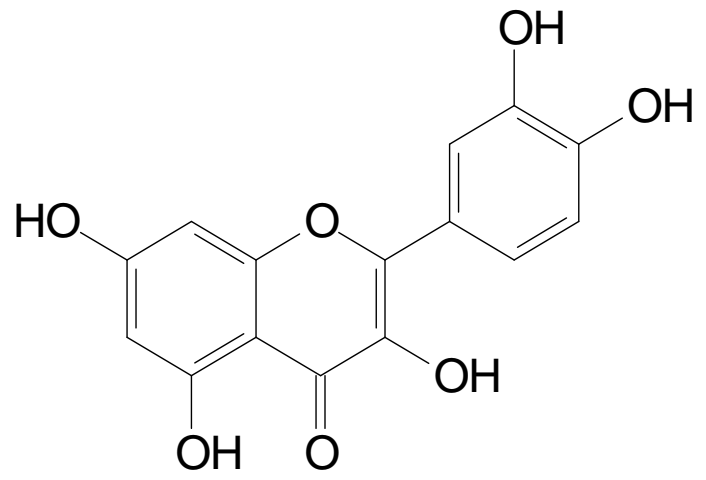

Fig. 1. Chemical structure of quercetin

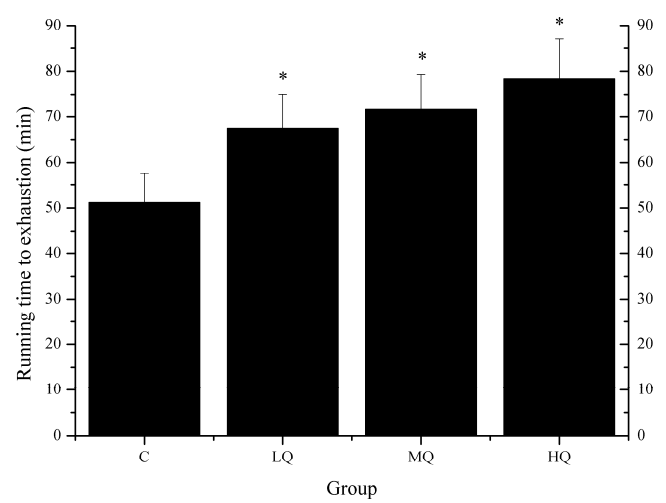

Fig. 2. Effects of quercetin on running time to exhaustion of rats. Data were expressed as means \pm SD of eight. ${ }^{*} \mathrm{p}<$ 0.05 , compared with control (C) group

Effects of quercetin on serum lactate dehydrogenase and creatine kinase levels of rats. Lactate dehydrogenase (LDH) and creatine kinase (CK) are non-plasma specific enzymes that are released into blood as the result of the destruction of the cell membrane by oxidative stress or tissue damage, as well as the direct destruction of the cell wall and tissue necrosis. Therefore, changes in the LDH and CK activity in blood can be used as an indirect index of muscle damage [7]. The increase in LDH and CK levels in the blood, which result from exhaustive exercise, have been well documented [8]. 
As shown in Fig. 3, compared to the C group, LQ, MQ and HQ groups showed a significant reduction in serum LDH and CK levels in liver $(\mathrm{p}<0.05)$. This data clearly reveal that quercetin could reduce muscle damage caused by exhaustive exercise.

Effects of quercetin on myeloperoxidase levels in liver and muscle of rats. It is also well known that exhaustive exercise causes severe damage to muscle cells and consequently stimulates an inflammatory response [9]. Many studies have demonstrated that exhaustive exercise leads to the release of myeloperoxidase (MPO) from neutrophils which then induces severe oxidative damage. MPO is regarded not only as an index of inflammation but also as an index of oxidative damage [10]. As shown in Fig. 4, compared to the $\mathrm{C}$ group, LQ, MQ and HQ groups showed a significant reduction in MPO levels in liver ( $<$ 0.05); MQ and HQ groups showed a significant reduction in MPO levels in muscle $(p<0.05)$, while LQ group was not significantly different was observed $(p>0.05)$. This data show that quercetin is able to prevent exercise-induced oxidative damage.

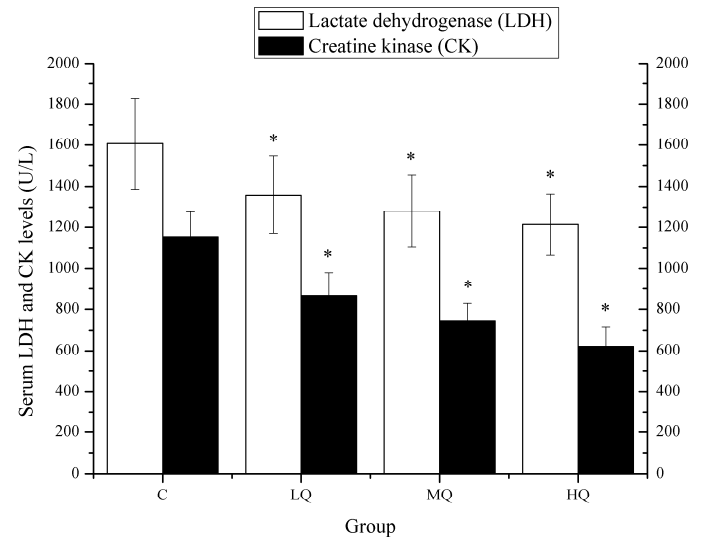

Fig. 3. Effects of quercetin on serum lactate dehydrogenase and creatine kinase levels of rats. Data were expressed as means \pm SD of eight. *p $<0.05$, compared with control (C) group group

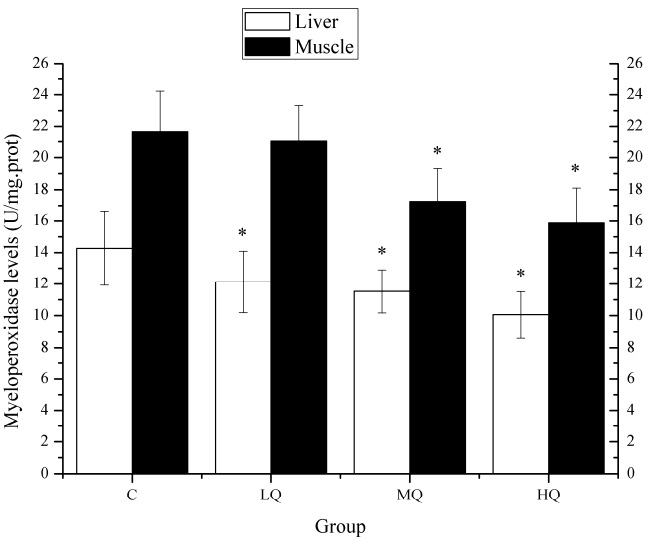

Fig. 4. Effects of quercetin on myeloperoxidase levels in liver and muscle of rats. Data were expressed as means \pm SD of eight. ${ }^{*} \mathrm{p}<0.05$, compared with control (C) group

Effects of quercetin on malondialdehyde levels in liver and muscle of rats. Exhaustive exercise-induced oxidative stress, leading to lipid peroxidation (LPO) has been well documented over the last decade [11]. It has been reported that LPO is involved in damage to specific mitochondrial proteins and transport systems by direct inhibition of enzymes, resulting in loss of mitochondrial integrity. LPO is thought to be a prominent and especially deleterious form of neuronal oxidative injury by damaging the neuronal membrane [12]. The MDA, a metabolite of phospholipid peroxidation, is a good indicator of the degree of LPO. Exercise has been shown to induce MDA levels and a recovery can be obtained by dietary antioxidants[13]. As shown in Fig. 5, the MDA levels in liver and muscle in the LQ, MQ and HQ groups were significantly lower than that of the $\mathrm{C}$ (control) group $(\mathrm{p}<0.05)$. This data clearly reveal that quercetin could prevent exercise-induced LPO, which is another confirmation that quercetin has a protective effect on exercise-induced oxidative damage.

Effects of quercetin on liver and muscle 8-hydroxy-2'-deoxyguanosine levels of rats. It is known that DNA oxidation is formed from hydroxyl radicals and singlet oxygen attack on 2'-deoxyguanosine, resulting in a hydroxyl moiety replacing the hydrogen atom [11]. Oxidative damage to DNA is generally measured by the levels of $8-\mathrm{OHdG}$, because it represents $5 \%$ of the total oxidized bases in the DNA and is present in quantities that are sufficient to be readily detected [14]. As shown in Fig. 6, compared to the $\mathrm{C}$ group, HQ groups showed a significant reduction in 8-OHdG levels in liver $(\mathrm{p}<0.05)$, while $L Q$ and MQ groups were not significantly different was observed ( $>$ 0.05); MQ and HQ groups showed a significant reduction in 8-OHdG levels in muscle $(\mathrm{p}<0.05)$, while LQ group was not significantly different was observed $(p>0.05)$. This data show that quercetin could reduce oxidative stress-induced DNA damage during exercise. 


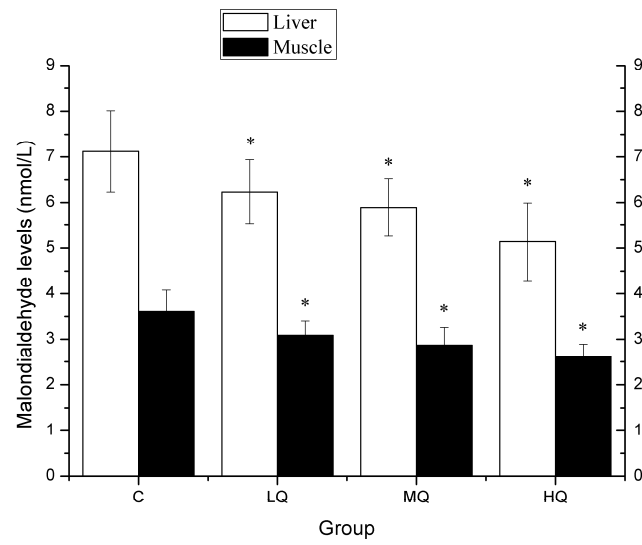

Fig. 5. Effects of quercetin on malondialdehyde levels in liver and muscle of rats. Data were expressed as means \pm SD of eight. *p $<0.05$, compared with control (C) group

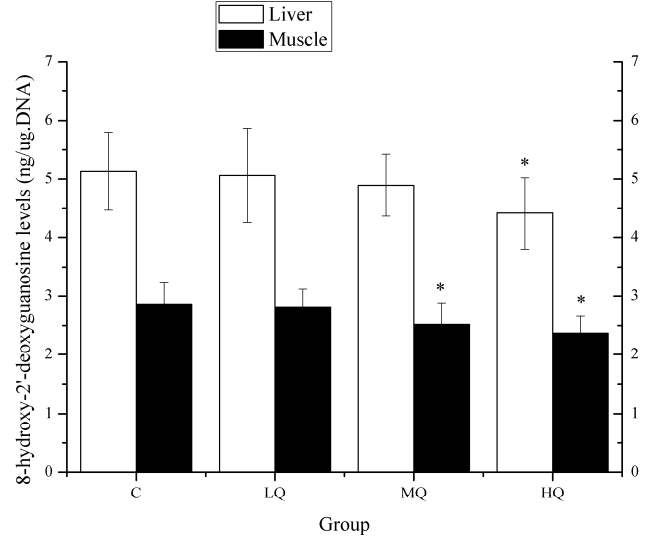

Fig. 6. Effects of quercetin on liver and muscle 8-hydroxy-2'-deoxyguanosine levels of rats. Data were expressed as means \pm SD of eight. ${ }^{*} \mathrm{p}<0.05$, compared with control (C) group

\section{Summary}

The present study clearly demonstrates that quercetin has a protective effect on exercise-induced oxidative damage, which prolonged the running time to exhaustion of rats, as well as decreasing $\mathrm{LDH}$, CK, MPO, MDA and 8-OHdG levels. As a result, it may be concluded that, probably due to its antioxidant effects. However, further study is needed to elucidate the exact mechanism of protective effect of quercetin on oxidative damage.

\section{References}

[1] H.N. Liu, Y. Liu, L.L. Hu, Y.L. Suo, L. Zhang, F. Jin and Y. Li: Poult Sci Vol. 93 (2014), p. 347.

[2] Y.S. Dong, J.L. Wang, D.Y. Feng, H.Z. Qin and C. Li: Int. J. Med. Sci Vol. 11 (2014), p. 282.

[3] J.Q. Ma, Z. Li, W.R. Xie, C.M. Liu and S.S. Liu: Int. Immunopharmacol Vol. 28 (2015), p. 531.

[4] S.F. Nabavi, G.L. Russo, M. Daglia and S.M. Nabavi: Food Chem Vol. 179 (2015), p. 305.

[5] H.Y. Lin, H. Feng and R. Putheti: Afr. J. Microbiol. Res Vol. 3 (2009), p. 774.

[6] C. Xu, J. Lv, Y.M. Lo, S.W. Cui, X. Hu and M. Fan: Carbohydr Polym Vol. 92 (2013), p. 1159.

[7] H.T. Kim and C.H. Chae: Biol. Sport Vol. 23 (2006), p. 143.

[8] E.S. Rawson, B. Gunn and P.M. Clarkson: J. Strength. Cond. Res Vol. 15 (2001), p. 178.

[9] A. Childs, C. Jacobs, T. Kaminski, B. Halliwell and C. Leeuwenburgh: Free Radic. Biol. Med Vol. 31 (2001), p. 745.

[10]C.C. Huang, T.J. Lin, Y.F. Lu, C.C. Chen and W.T. Lin: Chin. J. Physiol Vol. 52 (2009), p. 306.

[11]M. Bahman, R.N. Farhad, S. Zivar and R. Rahman: Kinesiology Vol. 45 (2013), p. 30.

[12] H.T. Hao: Trop. J. Pharm. Res Vol. 13 (2014), p. 1319.

[13] E. Síktar, D. Ekinci, E. Síktar, S. Beydemir, I. Gülçin and M. Günay: Eur. J. Pharmacol Vol. 668 (2011), p. 407.

[14]R. Rahimi: J. Strength Cond. Res Vol. 25 (2011), p. 3448. 